\title{
Can Doubly Strange Dibaryon Resonances be Discovered at RHIC?
}

\author{
S. D. Paganis,[] G. W. Hoffmann, R. L. Rayf], J.-L. Tang[ and T. Udagawa \\ Department of Physics \\ The University of Texas at Austin, Austin, Texas 78712
}

\begin{abstract}
R. S. Longacre
Physics Department, Brookhaven National Laboratory, Upton, NY 11973
\end{abstract}

\begin{abstract}
The baryon-baryon continuum invariant mass spectrum generated from relativistic nucleus + nucleus collision data may reveal the existence of doubly-strange dibaryons not stable against strong decay if they lie within a few $\mathrm{MeV}$ of threshold. Furthermore, since the dominant component of these states is a superposition of two color-octet clusters which can be produced intermediately in a color-deconfined quark-gluon plasma (QGP), an enhanced production of dibaryon resonances could be a signal of QGP formation. A total of eight, doubly-strange dibaryon states are considered for experimental search using the STAR detector (Solenoidal Tracker at RHIC) at the new Relativistic Heavy Ion Collider (RHIC). These states may decay to $\Lambda \Lambda$ and/or $p \Xi^{-}$, depending on the resonance energy. STAR's large acceptance, precision tracking and vertex reconstruction capabilities, and large data volume capacity, make it an ideal instrument to use for such a search. Detector performance and analysis sensitivity are studied as a function of resonance production rate and width for one particular dibaryon which can directly strong decay to $p \Xi^{-}$, but not $\Lambda \Lambda$. Results indicate that such resonances may be discovered using STAR if the resonance production rates are comparable to coalescence model predictions for dibaryon bound states.
\end{abstract}

To be published in Physical Review $C$

\footnotetext{
${ }^{1}$ Present address: Nevis Laboratories, Columbia University, P. O. Box 137, Irvington, NY 10533.

${ }^{2}$ Corresponding author: electronic address: ray@physics.utexas.edu

${ }^{3}$ Present address: Department of Physics, National Central University, Chung-Li, Taiwan 320.
} 


\section{Introduction}

It is generally assumed that quantum chromodynamics (QCD) is capable of describing the structure and spectroscopy of baryons and mesons. In addition, a number of models based on QCD predict other types of quark and gluonic systems such as pure gluonic states (glueballs), hybrids ( $q \bar{q} g)$, four quark di-mesons ( $q q \bar{q} \bar{q}$,

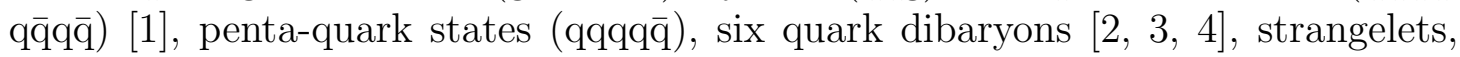
and for large systems several fm in size at sufficiently high temperature and/or net baryon density, the color-deconfined quark-gluon plasma (QGP). Extensive experimental searches have been made for all these systems.

In particular, many experiments $($ e.g. [3, 4, 5, 6, 7]) have searched for a stable (at least to strong decay), doubly-strange flavor-singlet dibaryon $\left(\mathrm{H}_{0}\right)$ of the six quark $\mathrm{SU}(3)$-flavor $\left[\mathrm{SU}(3)_{f}\right]$ multiplets $[8]$. This hypercharge $(Y)=0$ dibaryon was predicted by Jaffe [2] to be stable against strong decay, but not to weak decay; hence the experiments generally searched for characteristic weak decay topologies. So far, no conclusive evidence for a bound $\mathrm{H}_{0}$ has been presented [3, 4, 5, 6, 7]. However, the $\mathrm{H}_{0}$ mass may be larger than $M_{\Lambda \Lambda} \equiv M_{\Lambda}+M_{\Lambda}$, the $\Lambda \Lambda$ strong decay threshold [9, 10, 11]. In this case the $\mathrm{H}_{0}$ could be a strong interaction resonance. If the resonance lies only a few $\mathrm{MeV}$ above $M_{\Lambda \Lambda}$, then it may be possible to see experimental evidence for its existence [9]. In addition, it is important to point out that several other doubly-strange dibaryon states occur in the $\mathrm{SU}(3)_{f}$ multiplets which do not directly strong decay to $\Lambda \Lambda$ and which may also appear as resonances in the $\Lambda \Lambda$ and $N \Xi$ systems.

Experimental searches for a stable $\mathrm{H}_{0}$ have attempted to create and observe the $\mathrm{H}_{0}$ through: (1) double-strangeness-exchange reactions, such as $A\left(K^{-}, K^{0}\right) X$, $A\left(K^{-}, K^{+}\right) X$; (2) $\Xi^{-} A$ capture [5] on nuclear targets with $A \geq 2$; and (3) proton + nucleus [6, [] or nucleus + nucleus collisions where the $\mathrm{H}_{0}$ production mechanism could proceed via multi-hyperon production followed by coalescence [12, 13, 14, 15]. Reactions such as $d\left(K^{-}, K^{0}\right) \mathrm{H}_{0}$, for example, produce a relatively clean final state but suffer from poor momentum matching and subsequently, a reduced production rate. Heavy ion collisions, although producing a complex final state, generate a large number of co-moving hyperons and non-strange baryons from which dibaryons could either coalesce or scatter through resonances. The invariant mass spectrum for $\Lambda \Lambda$ pairs produced in $158 \mathrm{~A} \mathrm{GeV} / \mathrm{c} \mathrm{Pb}+\mathrm{Pb}$ collisions [data from WA97 [16]] does not reveal any resonance like structures. However, the data are too coarsely binned and lack sufficient statistics to be relevant for the type of experimental search proposed here. On the other hand, a three standard deviation enhancement above background was observed in the $\Lambda \Lambda$ invariant mass spectrum

\footnotetext{
${ }^{4}$ In the meson sector nature provides several examples of narrow states that lie a few $\mathrm{MeV}$ above their strong decay thresholds. The $\phi(1020), D^{*}(2010)^{ \pm}, D^{*}(2007)^{0}$ and $D_{\mathrm{S} 1}(2536)^{+}$ mesons are 32.1, 5.8, 7.1 and $35.0 \mathrm{MeV}$, respectively, above their strong decay thresholds and have total widths of $4.4,<0.13,<2.1$ and $<2.3 \mathrm{MeV}$, respectively. The latter three quantities represent upper limits at the $90 \%$ confidence level.
} 
from threshold to $30 \mathrm{MeV}$ above threshold, in the $\Lambda \Lambda$ pair production data from the ${ }^{12} \mathrm{C}\left(K^{-}, K^{+}\right)$reaction [17].

The Relativistic Heavy Ion Collider (RHIC) facility at the Brookhaven National Laboratory, together with four new detectors, is beginning a program of research whose primary goal is to produce a quark-gluon plasma and study its properties. It is noteworthy that a color-deconfined QGP could enhance significantly the production of $\mathrm{H}_{0}$ dibaryons compared to the production expected from a hot hadronic gas [13]. The reason is that the largest component of the six quark dibaryon wave function consists of a color-singlet superposition of color-octet-octet components [9, 13, 18]:

$$
|H\rangle=\sqrt{\frac{1}{5}}|B B\rangle+\sqrt{\frac{4}{5}}\left|8_{c} \otimes 8_{c}\right\rangle .
$$

Here, $\left|8_{c} \otimes 8_{c}\right\rangle$ represents the color-octet-octet components, and $|B B\rangle$ denotes a summation of states composed of two physical baryons. Enhanced dibaryon production can occur by way of fusion of color-octet clusters within the deconfined plasma [13]. If evidence for dibaryon resonances is observed in the data from RHIC collisions, then analyses of such data would provide an independent basis for studying the QGP.

In this work we explore the feasibility of observing evidence for the existence of several doubly-strange dibaryon resonances through study of the $\Lambda \Lambda$ and $N \Xi$ invariant mass spectra constructed from relativistic heavy ion collision data to be taken at RHIC.] We focus on using the STAR [19] detector [with the Silicon Vertex Tracker (SVT) and Silicon Strip Detector (SSD) [20] upgrades] since it is the most suited of the RHIC detectors for such a program. A preliminary study [20, 21] suggested that STAR could be used to search for $\mathrm{H}_{0} \rightarrow \Lambda \Lambda$ with resonance masses a few hundred $\mathrm{MeV}$ above $M_{\Lambda \Lambda}$. The focus of the present work is on the $N \Xi$ decay channel with resonance masses a few $\mathrm{MeV}$ above the strong decay threshold.

In Sec. 2 the doubly-strange dibaryons and their possible decay schemes are discussed. Using the P-matrix formalism [9] the resonance widths are estimated in Sec. 3. In Sec. 1 the $J^{\pi}=0^{+}, \mathrm{I}($ isospin $)=1, \mathrm{I}_{3}=0$ dibaryon member of the $\mathrm{SU}(3)_{f}$ 27-plet [8] [referred to as $\mathrm{H}^{27}\left(J^{\pi}, \mathrm{I}, \mathrm{I}_{3}\right)$ with $J^{\pi}=0^{+}, \mathrm{I}=1$ and $\mathrm{I}_{3}=0$ ], which cannot directly strong decay to $\Lambda \Lambda$, is used to explore the statistical aspects and sensitivity of analysis of STAR data to the existence of such resonances, if they exist. A new, fast simulation detector response code (FSDR) is used for this study. Finally, a summary and conclusions are presented in Sec. 5 .

\footnotetext{
${ }^{5}$ Formation of hyper-nuclear baryon-baryon bound states or slightly unbound states is also possible (e.g. strange baryon analogs of the deuteron and the nucleon + nucleon ${ }^{1} \mathrm{~S}_{0}$ unbound state). Such states, if they exist, are expected to produce broad enhancements over many tens of $\mathrm{MeV}$ in the $\Lambda \Lambda$ or $N \Xi$ spectra, and not the narrow resonance structures predicted for six quark states which are the focus of the present work.
} 


\section{Dibaryon States}

The direct product space of the $J^{\pi}=\frac{1}{2}^{+}$baryon octet with itself can be written in terms of irreducible representations of $\mathrm{SU}(3)_{f}$ [8]:

$$
\mathbf{8} \otimes \mathbf{8}=\mathbf{1} \oplus \mathbf{8} \oplus \mathbf{8} \oplus \mathbf{1 0} \oplus \overline{\mathbf{1 0}} \oplus \mathbf{2 7} .
$$

The hypercharge $(Y)$ ranges from $+2(\mathrm{NN}$, nucleon-nucleon) to $-2(\Xi \Xi)$ for these dibaryon states. The $Y=2$ members include the deuteron of the $\overline{\mathbf{1 0}}$-multiplet. The $Y=1$ states contain $\mathrm{N} \Lambda$ and $\mathrm{N} \Sigma$ components. Sharp enhancements in the $\mathrm{N} \Lambda$ spectra at $2129 \mathrm{MeV}$ [22] [the $\mathrm{N} \Sigma$ threshold [23, 24]] and $2139 \mathrm{MeV}$ [23] have been observed in single strangeness-exchange reactions such as $\mathrm{d}(K, \pi) \mathrm{X}$ and $\mathrm{d}(\pi, K) \mathrm{X}$. The $Y=-1$ and -2 states contain the experimentally difficult $\Xi \Lambda, \Xi \Sigma$ and $\Xi \Xi$ components. Finally, the $Y=0$ dibaryons contain $\Lambda \Lambda, N \Xi, \Lambda \Sigma$, and $\Sigma \Sigma$ components. Since $N \mathrm{~s}, \Lambda \mathrm{s}, \Sigma \mathrm{s}$, and $\Xi \mathrm{s}$ are produced in relativistic heavy ion $A+A$ central collisions, the RHIC experiments will provide an excellent opportunity to search for some of these $Y=0$ dibaryons.

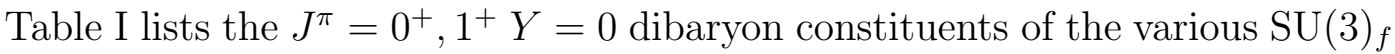
multiplets in Eq. (2) from Refs. [2, 8] along with the dominant decay modes for a wide range of assumed dibaryon masses. The mass limits where strong and/or electromagnetic (EM) decay channels open are noted for each state. In general the best mass ranges to explore for resonances are those lying just above the strong and/or EM decay thresholds in Table —. Jaffe [2] predicted a significant increase in the dibaryon masses with increasing dimensionality of the $\mathrm{SU}(3)_{f}$ representation. However, more recent calculations based on QCD sum rules [25, 26] indicate that the $Y=0$ dibaryons in different multiplets should be similar in mass; the principal mass dependence of the $\mathbf{8} \otimes \mathbf{8}$ dibaryons is due to the explicit $\mathrm{SU}(3)_{f}$ symmetry breaking caused by the strange quark mass. All of the $Y=0$ dibaryon states in Table $[$ will be considered in this work, since at present there is no reason to exclude them from experimental searches.

For masses between $M_{\Lambda \Lambda}$ and $M_{N \Xi}$ the singlet $\mathrm{H}_{0}$ and $\mathrm{H}^{27}\left(0^{+}, 0,0\right)$ can strong decay to $\Lambda \Lambda$. Recently, using a P-matrix formalism, Bashinsky and Jaffe [9] considered a hypothetical singlet $\mathrm{H}_{0}$ strong-decay resonance of mass several $\mathrm{MeV}$ above the $\Lambda \Lambda$ (strong decay) threshold. For a variety of assumptions, they found that, owing to kinematic effects near threshold, the cross sections for S-wave $\Lambda \Lambda$ elastic scattering should show structures whose scales are of the order of several $\mathrm{MeV}$. It may be that the $\Lambda \Lambda$ invariant mass spectrum generated from relativistic heavy ion collision data will show similar structure that can be taken as evidence of the existence of such a resonance. The analysis in [9] also applies for the $\mathrm{H}^{27}\left(0^{+}, 0,0\right)$ case.

\footnotetext{
${ }^{6}$ It is also worth noting that the $Y=2$ deuteron member of the $\overline{\mathbf{1 0}}$-flavor multiplet and the nucleon + nucleon ${ }^{1} \mathrm{~S}_{0}$ unbound state member of the $\mathbf{2 7}$-flavor multiplet differ in mass by only a few MeV. Calculations [24] based on a SU(3) invariant pseudoscalar and vector meson exchange model for the baryon-baryon system predict the $0^{+} \mathbf{2 7}$-plet dibaryon mass to be less than the mass of the singlet $\mathrm{H}_{0}$.
} 
The 8-, 10- and $\overline{\mathbf{1 0}}$-plet $J^{\pi}=1^{+}$dibaryons and the $\mathrm{H}^{27}\left(0^{+}, 1, \mathrm{I}_{3}\right)$ and $\mathrm{H}^{27}\left(0^{+}, 2, \mathrm{I}_{3}\right)$ dibaryons cannot strong decay to $\Lambda \Lambda$ since antisymmetrization requires the final $\Lambda \Lambda$ system to be spin-singlet-even or spin-triplet-odd and $\mathrm{I}(\Lambda \Lambda)=0$. The $\mathrm{H}^{27}\left(0^{+}, 1,0\right)$ and $\mathrm{H}^{27}\left(0^{+}, 2,0\right)$ dibaryons can, however, strong decay to $\Lambda \Lambda$ by way of the small $\mathrm{I}=0$ isospin admixture which contributes to the physical states due to Coulomb induced isospin mixing, but this transition rate should be much less than that for $\mathrm{H}_{0} \rightarrow \Lambda \Lambda$. The four $J^{\pi}=1^{+}, \mathrm{I}_{3}=0$ states can EM decay to $\Lambda \Lambda \gamma$ via E1 transitions leaving the $\Lambda \Lambda$ in relative ${ }^{3} \mathrm{P}_{J}$ states. The $\mathrm{H}^{27}\left(0^{+}, 1,0\right)$ and $\mathrm{H}^{27}\left(0^{+}, 2,0\right)$ states can also EM decay to $\Lambda \Lambda \gamma$, for example via E1 transition to the ${ }^{3} \mathrm{P}_{1} \Lambda \Lambda$ final state. All remaining states (i.e., those with $\mathrm{I}_{3} \neq 0$ ) in this mass range (from $M_{\Lambda \Lambda}$ to $M_{N \Xi}$ ) can decay only weakly. It is likely that the $J^{\pi}=1^{+}$ states are of larger mass than the $0^{+}$states and thus even more difficult to deal with experimentally.

In the mass range from $M_{N \Xi}$ to $M_{\Sigma \Sigma}$ all states with $\mathrm{I}<2$ decay strongly to $\Lambda \Lambda, N \Xi$ or $\Lambda \Sigma$ as indicated in Table 【. The $\mathrm{H}^{27}\left(0^{+}, 2, \pm 1\right)\left[\mathrm{H}^{27}\left(0^{+}, 2,0\right)\right]$ states can strong decay to $N \Xi$ and $\Lambda \Sigma[N \Xi, \Lambda \Sigma$ and $\Lambda \Lambda]$ via isospin admixtures or they can decay electromagnetically. The $\mathrm{H}^{27}\left(0^{+}, 2, \pm 2\right)$ remain blocked to both strong and EM decays up to $M_{\Sigma \Sigma}$ due to charge conservation. The $\mathrm{H}^{27}\left(0^{+}, 1, I_{3}\right)$ members of the 27-multiplet cannot strong decay to $\Lambda \Lambda$ because of isospin conservation; however, they can strong decay to $N \Xi$ and $\Lambda \Sigma$ if the mass is greater than $M_{N \Xi}$ and $M_{\Lambda \Sigma}$, respectively. Above $M_{\Sigma \Sigma}$ all states in Table [ can decay strongly.

Table [1] lists the optimum decay channels and mass ranges for possible $Y=0$ dibaryon resonance searches. The last column indicates whether the channels include those appropriate for STAR [19]. Of the listed decay products, STAR can identify only protons (p), $\Lambda$ s and $\Xi^{-}$s. Eight of the $Y=0$ dibaryon states in Tables $\mathbb{\text { I and }}$ (corresponding to each of the $\mathrm{I}_{3}=0$ states) are seen to be appropriate for a program of research with STAR.

Evidence for the $\mathrm{H}_{0}$ and $\mathrm{H}^{27}\left(0^{+}, 0,0\right)$ states might be seen in the $\Lambda \Lambda$ invariant mass spectrum within several $\mathrm{MeV}$ of threshold. The four $J^{\pi}=1^{+}, \mathrm{I}_{3}=0$ states, if in the mass range from $M_{\Lambda \Lambda}$ to $M_{n \Xi^{0}}$, will decay electromagnetically to $\Lambda \Lambda \gamma$. If the photon is not detected, the $\Lambda \Lambda$ invariant mass spectrum would appear as a broad continuum. However, an enhancement still might be observed if the resonance masses are only a few $\mathrm{MeV}$ above $M_{\Lambda \Lambda}$. These four resonances might also be observed in the $p \Xi^{-}$spectrum if either lies just above the $M_{p \Xi^{-}}=2259.6 \mathrm{MeV}$ strong breakup threshold. 1

If the resonance mass exceeds $M_{\Lambda \Lambda}$, the $\mathrm{H}^{27}\left(0^{+}, 1,0\right)$ and $\mathrm{H}^{27}\left(0^{+}, 2,0\right)$ states can decay either electromagnetically to $\Lambda \Lambda \gamma$ or strongly via the small $\mathrm{I}=0$ admixture. The competition between these branches determines the experimental signature. If the strong interaction, isospin admixture decay dominates the EM decay, then the $\mathrm{I}=1(2)$ state might be observed if its mass is between $M_{\Lambda \Lambda}$ and $M_{n \Xi^{0}}\left(M_{\Sigma^{0} \Sigma^{0}}\right)$.

\footnotetext{
${ }^{7}$ Due to Coulomb interactions the $n \Xi^{0}$ and $p \Xi^{-}$mass thresholds are split by $5.1 \mathrm{MeV}$. Strong decay to $N \Xi$ therefore opens at $M_{n \Xi^{0}}=2254.5 \mathrm{MeV}$, whereas the observable decay channel for STAR, dibaryon $\rightarrow p \Xi^{-}$, does not open until $2259.6 \mathrm{MeV}$.
} 
If the EM decay dominates, then both $\mathrm{I}=1$ and 2 states might only be observed as enhancements in the continuum just above $M_{\Lambda \Lambda}$. Otherwise the $\mathrm{H}^{27}\left(0^{+}, 1,0\right)$ state might be seen in the $p \Xi^{-}$invariant mass spectrum just above the $M_{p \Xi^{-}}$ threshold. In this case $\mathrm{H}^{27}\left(0^{+}, 1,0\right) \rightarrow p \Xi^{-}$might be observable with STAR. The $\mathrm{H}^{27}\left(0^{+}, 2,0\right)$ resonance could strong decay to $\Sigma^{0} \Sigma^{0}$ and $\Sigma^{+} \Sigma^{-}$final states, but the $\Sigma$ s, unfortunately, cannot be reconstructed by STAR.

In this initial simulation study we prefer to consider the dibaryon resonance with smallest angular momentum which may strong decay to $p \Xi^{-}$but not to $\Lambda \Lambda$, namely $\mathrm{H}^{27}\left(0^{+}, 1,0\right)$. The reason for this choice is the expectation that the combinatoric background contribution to the $p \Xi^{-}$invariant mass spectrum will be less than that for $\Lambda \Lambda$ for the case of RHIC data for $\mathrm{Au}+\mathrm{Au}$ central collisions (see Sec. 4.) Simulation studies for the remaining dibaryon resonances in Table [1] will be done in the future.

It is worth noting that threshold-cusp effects [23, 24, 27] could produce enhancements in the $\Lambda \Lambda$ spectra at the $N \Xi, \Sigma \Sigma$, etc., thresholds, independent of any possible resonances. This is also true for the $N \Xi$ spectra ( $\mathrm{I}=1$ component) at the $\Lambda \Sigma$ and $\Sigma \Sigma$ thresholds. Such enhancements are due to coupled-channels scattering effects near inelastic thresholds and must be treated carefully when searching for experimental evidence of dibaryon resonances.

Resonances occuring between the $n \Xi^{0}$ and $p \Xi^{-}$thresholds will decay primarily to $n \Xi^{0}$, although decay to $p \Xi^{-}$will occur if the resonance width is such that the distribution extends above $M_{p \Xi^{-}}$. For resonance masses near or a few $\mathrm{MeV}$ above $M_{p \Xi^{-}}$, the branching ratio between the $n \Xi^{0}$ and $p \Xi^{-}$channels depends upon the dynamics of the $n \Xi^{0}-p \Xi^{-}$-dibaryon coupled channels system, as well as on the kinematic density of final states factor. For resonance energies much above $M_{p \Xi^{-}}$the branching ratio is determined mainly by isospin invariance which results in a 50\% decay fraction for both channels. Thus the study in Sec. 1 will be in terms of the dibaryon resonance width and production rate for the observable dibaryon $\rightarrow p \Xi^{-}$decay channel only. Inclusion of the model-dependences associated with the $n \Xi^{0}-p \Xi^{-}$-dibaryon system lies well beyond the scope and purpose of this work.

Finally, we note that the decay products of resonance states formed deep within the interior of the collision region will undergo strong, final state interactions (FSI) so that observation of these resonances becomes more difficult. It is likely that only resonances occuring in the periphery of the QGP or in the mixed QGP-hadronic phase prior to freeze-out will lead to experimental signatures in invariant mass spectra. Detailed calculations of FSI effects on this and other potential QGP signals remain to be done.

\section{Dibaryon Resonance Widths}

Bashinsky and Jaffe 90 used the P-matrix scattering formalism to compute the $\Lambda \Lambda$ elastic scattering S-wave total cross section in the presence of a flavor-singlet, 
$\mathrm{H}_{0}$ dibaryon resonance located a few $\mathrm{MeV}$ above the $\Lambda \Lambda$ threshold. Their model is used in this section for the similar case of a possible $\mathrm{H}^{27}\left(0^{+}, 1,0\right)$ (for brevity referred to as $\mathrm{H}^{27}$ in the remainder of this paper) dibaryon resonance lying just above the $N \Xi$ threshold.

The total cross section for $N \Xi$ elastic scattering is given by

$$
\sigma_{\mathrm{TOT}}=\frac{4 \pi}{k_{1}} \operatorname{Im}\left(f_{11}\right),
$$

where $f_{11}$ is approximated by the forward, S-wave elastic scattering amplitude, which in the P-matrix formalism is written as [9]

$$
f_{11}=\frac{1}{\frac{1}{K_{1}^{(r e d)}}-i k_{1}} .
$$

The reduced K-matrix from Ref. [9] as a function of total energy $(\epsilon)$ in the $N \Xi$ channel is

$$
K_{1}^{(r e d)}(\epsilon) \simeq-\bar{a}_{1}^{\prime}-\frac{\rho_{1}^{\prime} / 2 m}{\epsilon-\epsilon_{t h 1}-\epsilon_{1}^{\prime}},
$$

where,

$$
\bar{a}_{1}^{\prime}=\frac{\bar{a}_{1}}{1-\rho_{1} b_{0}}, \rho_{1}^{\prime}=\rho_{1} \frac{1-\rho_{1} b_{0}+2 m \epsilon_{1} \bar{a}_{1} b_{0}}{\left(1-\rho_{1} b_{0}\right)^{2}}, \epsilon_{1}^{\prime}=\frac{\epsilon_{1}}{1-\rho_{1} b_{0}} .
$$

In Eqs. (河)-(的), $k_{1}$ is the relative center-of-momentum system wave number, $m$ is the reduced mass of the two-baryon $(N \Xi)$ system, $\epsilon_{t h 1}$ is the $N \Xi$ threshold energy, $\epsilon_{1}=\epsilon_{r}-\epsilon_{t h 1}$, and $\epsilon_{r}$ is the assumed resonance mass of the $\mathrm{H}^{27}$ dibaryon. Parameter $b_{0}$ is a resonance size parameter with typical value $(150 \mathrm{MeV})^{-1}, \rho_{1}$ is the resonance width parameter which is a function of the resonance state - decay channel coupling, and $\bar{a}_{1}$ is the $N \Xi$ scattering length which was assumed to be $(200 \mathrm{MeV})^{-1}$.

Elastic total cross section results obtained using Eqs. (3)-(6) are shown in Fig. 1 . by the solid lines for assumed values of $\epsilon_{1}=2,3$ and $5 \mathrm{MeV}$ and for the typical resonance width parameter $\rho_{1}=50 \mathrm{MeV}$. The unitarity limit, $4 \pi / k_{1}^{2}$, is indicated by the dashed lines. These results indicate that the resonance distribution displays a characteristic width roughly proportional to the difference between the resonance and threshold energies.

Resonances decaying to $p \Xi^{-}$may also decay to $n \Xi^{0}$ which is $5.1 \mathrm{MeV}$ lower in energy. These results suggest that resonances near $M_{p \Xi^{-}}$would be about $5 \mathrm{MeV}$ wide. It is reasonable to assume, however, that the $p \Xi^{-}$spectra for such cases would display a narrower range of enhancement because of the kinematic limit which cuts off the lower energy portion of the $p \Xi^{-}$invariant mass distribution. 


\section{Search for Dibaryon Resonances using STAR}

An environment conducive to the formation of dibaryon resonances may be the hot, dense nuclear matter that will be created through central $\mathrm{Au}+\mathrm{Au}$ collisions at RHIC. The experimental objective, then, is to infer evidence for the existence of such resonances through examination of $\Lambda \Lambda$ or $p \Xi^{-}$invariant mass spectra. Since the resonance cross sections are expected to be small, a large acceptance detector with the ability to do precision tracking to within a few $\mathrm{cm}$ of the production vertex is needed. In addition, the detector must handle high data rates and have event processing capabilities for very large data volumes. The STAR detector 19] at RHIC with its central vertex tracking system (SVT-SSD) is well-suited for such experiments. For the simulations to be discussed here, the parameters in Table [II] were used to approximate the kinematic acceptance and reconstruction performance of STAR (including the SVT-SSD). The momentum resolution and track reconstruction efficiencies were taken from previous simulation studies [20, 28.

Coalescence model calculations 13, 15 for $\mathrm{Au}+\mathrm{Au}$ central collisions at AGS energies ( $\sim 11 \mathrm{~A} \cdot \mathrm{GeV}$ fixed target) predict a wide range of production rates for the flavor-singlet $\mathrm{H}_{0}$ from of order $10^{-4}$ to 0.5 per event, depending on the assumed temperature and strangeness content of the hadron gas and on the assumed $\Lambda \Lambda$ interaction model. Color-deconfinement in the QGP could enhance the production rate via the color-octet-octet component of the dibaryon wave function [Eq. (四)]. At RHIC energies increased strangeness production and possible QGP formation could further enhance the dibaryon production rate above this range. Source expansion and collective flow in RHIC collisions could also affect coalescence model

predictions [14]. Comprehensive calculations including all these effects have not been done. Nevertheless, the results of such calculations suggest the necessary level of sensitivity for the experimental search to be meaningful.

In the following, an analytical model is presented which relates the statistical significance of the dibaryon resonance signal to the number of $\mathrm{Au}+\mathrm{Au}$ central RHIC collision events analyzed and the assumed resonance production rate and width. This is followed by a description of a fast numerical simulation model and presentation of statistical and sensitivity results for experimental detection of a hypothetical, narrow dibaryon resonance in the $p \Xi^{-}$channel just above threshold.

\subsection{Analytical Model}

The integrated signal for the $\mathrm{H}^{27} \rightarrow p \Xi^{-}$invariant mass distribution can be approximated by

$$
S \cong \sum_{i=1}^{N_{e v}} n_{H}^{i} e_{p} e_{\Xi} f_{H-c u t}=n_{H} e_{p} e_{\Xi} f_{H-c u t} N_{e v} \equiv \bar{S} N_{e v}
$$


where $n_{H}^{i}$ is the assumed number of dibaryon resonances in the $i^{\text {th }} \mathrm{Au}+\mathrm{Au}$ central collision event that decay to $p \Xi^{-}$with all daughter products going fully into the kinematic acceptance range specified in Table III, and $n_{H}$ is the average number of $\mathrm{H}^{27} \rightarrow p \Xi^{-}$decays per event for the data sample consisting of $N_{e v}$ events. Also in Eq. (7) $e_{p}$ and $e_{\Xi}$ are the proton and $\Xi^{-}$reconstruction efficiencies, respectively (number correctly reconstructed and accepted divided by number in the kinematic acceptance or detector fiducial volume), $f_{H-c u t}$ is the fraction of reconstructed $\mathrm{H}^{27} \rightarrow p \Xi^{-}$resonance decays that survive any remaining analysis cuts (e.g. cuts to remove fluctuations from the background subtracted spectra and the upper/lower $p \Xi^{-}$invariant mass limits), and $\bar{S}$ is the average signal per event [29]. The total background underneath the signal peak is approximated by

$$
B \cong \sum_{i=1}^{N_{e v}}\left(N_{p}^{i} N_{\Xi}^{i}-n_{H}^{i} e_{p} e_{\Xi}\right) F\left(\rho_{1}\right) \equiv \bar{B} N_{e v}
$$

where $N_{p}^{i}$ and $N_{\Xi}^{i}$ represent the number of reconstructed and accepted proton and $\Xi^{-}$candidates for the $i^{\text {th }}$ event, $F\left(\rho_{1}\right)$ represents the relative fraction of random $p \Xi^{-}$pairs whose invariant mass occurs within the domain of the $\mathrm{H}^{27}$ mass peak, and $\bar{B}$ is defined as the average background per event. The fraction, $F\left(\rho_{1}\right)$, depends on the proton and $\Xi^{-}$momentum space distributions for the collision events (after cuts) and on the $p \Xi^{-}$invariant mass range which in turn depends on the width of the resonance peak.

The numbers of candidate protons and $\Xi^{-}$include several contributions which are represented by the following:

$$
\begin{aligned}
& N_{p}^{i}=\left(n_{H}^{i}+n_{H, p}^{i}+n_{t h, p}^{i}\right) e_{p}+N_{\text {False }, p} \\
& N_{\Xi}^{i}=\left(n_{H}^{i}+n_{H, \Xi}^{i}+n_{t h, \Xi}^{i}\right) e_{\Xi}+N_{\text {False }, \Xi},
\end{aligned}
$$

where $n_{H, p}^{i}\left(n_{H, \Xi}^{i}\right)$ is the number of protons $\left(\Xi^{-} \mathrm{s}\right)$ for the $i^{t h}$ event produced by $\mathrm{H}^{27}$ resonance decays for which the proton (all decay products of the $\Xi^{-}$) entered the detector acceptance but all decay products of the $\Xi^{-}$(the proton) did not. In Eqs. (9) and (10) $n_{t h, p}^{i}$ and $n_{t h, \Xi}^{i}$ represent the remaining number of protons and $\Xi^{-} \mathrm{s}$ produced in the collision, for example by hadronization and/or rescattering processes, which enter the detector acceptance. The quantities $N_{\text {False, } p}$ and $N_{\text {False, } \Xi}$ represent the number of incorrectly identified and accepted protons and $\Xi^{-} \mathrm{s}$ per event. The numbers of proton and $\Xi^{-}$contaminants depend on various analysis cuts, overall event multiplicity, etc. In general, tighter cuts in the reconstruction and analysis result in lower efficiency and reduced contamination, while relaxed cuts have the opposite effects. The efficiencies and contaminants per event in this section are averages of inclusive quantities.

The total number of counts, or yield $(Y)$, for the invariant mass spectra in the domain of the $\mathrm{H}^{27}$ resonance peak for $N_{e v} \mathrm{Au}+\mathrm{Au}$ collision events is

$$
Y=S_{T}+B=S+S^{\prime}+B=\left(\bar{S}+\bar{S}^{\prime}+\bar{B}\right) N_{e v}
$$


where

$$
S_{T}=\sum_{i=1}^{N_{e v}} n_{H}^{i} e_{p} e_{\Xi}=n_{H} e_{p} e_{\Xi} N_{e v}=S+S^{\prime}
$$

which defines $S^{\prime}$. The total error in the signal is assumed to be a sum of statistical and systematic errors given by

$$
\Delta S=\Delta S_{\text {stat }}+\Delta S_{\text {syst }}
$$

where the statistical error in $S$ is determined by the independent statistical errors in the total yield $(\Delta Y)$, background $(\Delta B)$, and $S^{\prime}\left(\Delta S^{\prime}\right)$, where

$$
\Delta S_{\text {stat }}=\sqrt{(\Delta Y)^{2}+\left(\Delta S^{\prime}\right)^{2}+(\Delta B)^{2}}=\sqrt{S+2\left(B+S^{\prime}\right)} .
$$

The systematic error in $S$ is assumed to be proportional to $N_{e v}$, where $\Delta S_{\text {syst }}=$ $\Delta \bar{S}_{\text {syst }} N_{e v}$.

The statistical significance of the observed resonance peak can be expressed as the ratio of signal to signal error, which in the present model is given by

$$
\frac{S}{\Delta S}=\frac{\bar{S} N_{e v}}{\sqrt{\bar{S}+2\left(\bar{B}+\bar{S}^{\prime}\right)} \sqrt{N_{e v}}+\Delta \bar{S}_{s y s t} N_{e v}} .
$$

If $f_{H-c u t}$ is constant [29] and systematic errors are negligible, $S / \Delta S$ increases with $\sqrt{N_{e v}}$ as expected, whereas for finite $\Delta \bar{S}_{s y s t}, S / \Delta S$ is limited to $\bar{S} / \Delta \bar{S}_{\text {syst }}$ for many events.

Solving Eq. (15) for $N_{e v}$ yields (assuming constant $f_{H-c u t}$ [29])

$$
N_{e v}=\frac{(S / \Delta S)^{2}\left[\bar{S}+2\left(\bar{B}+\bar{S}^{\prime}\right)\right]}{\left[\bar{S}-(S / \Delta S) \Delta \bar{S}_{\text {syst }}\right]^{2}}
$$

which provides an estimate of the number of collision events which must be obtained and analyzed in order to achieve a specified value of $S / \Delta S$. Systematic errors limit the detection capability such that

$$
\bar{S}>(S / \Delta S) \Delta \bar{S}_{\text {syst }},
$$

which results in a minimum, detectable dibaryon resonance production rate given by,

$$
n_{H}>\frac{(S / \Delta S) \Delta \bar{S}_{\text {syst }}}{e_{p} e_{\Xi} f_{H-c u t}},
$$

assuming the required number of events from Eq. (16) can be achieved.

Optimization of a particular experiment and data analysis program (e.g. number of events and cut parameters) can be guided by the results presented in this section provided a reasonable estimate of systematic errors can be made. The latter must wait until experience is gained with analysis of actual STAR data and therefore lies well beyond the scope of the present study. The remaining discussion and results focus on the statistical requirements for detecting $\mathrm{H}^{27} \rightarrow p \Xi^{-}$. In the following, Eqs. (15) and (16) were used to estimate the dependence of $(S / \Delta S)$ on $N_{e v}$ and $n_{H}$. 


\subsection{Numerical Simulations}

A fast simulation detector response code (FSDR) was developed to provide rapid evaluation of the capabilities of relativistic heavy ion experiments with respect to proposed new measurements and research programs. In simplest terms, FSDR projects a given input list of particles from an event generator directly to the final, reconstructed particle list. Specific detector acceptance and track reconstruction performance parameters are supplied from realistic simulations and analyses. The values assumed for STAR are listed in Table [1] [20, 28, 30]. FSDR propagates (freely) and decays unstable particles such as $\Lambda$ and $\Xi^{-}$according to known branching ratios and lifetimes and applies acceptance cuts, track finding efficiencies, particle identification efficiencies, and momentum resolution smearing to the charged primary particles and to the charged daughter particles from the decays.

Following this, FSDR reconstruction of $\Lambda$ and $\Xi^{-}$is very similar to that used in the actual STAR reconstruction analysis. The charged particles selected for this analysis were assumed to be reconstructed in both the SVT-SSD and TPC (Time Projection Chamber). The SVT-SSD provides excellent track position resolution (few tens of microns) [20, 28, 30] in the decay region within several $\mathrm{cm}$ of the primary vertex. Reconstructed trajectories (assumed to be helices) were projected to the primary vertex where an impact parameter cut $(1 \mathrm{~mm})$ differentiated primary particles (i.e., those assumed to emerge from the primary collision vertex) from secondary particles (i.e., those assumed to come from decay vertices). Candidate $\Lambda$ decays were found by a distance of closest approach (DCA) cut (2 mm) among the projected trajectories of the secondary protons and $\pi^{-} \mathrm{s}$. The resulting $\Lambda$ reconstruction in the $p \pi^{-}$invariant mass spectrum is shown in Fig. 2 for 600 central $\mathrm{Au}+\mathrm{Au}$ HIJET [31] collision events which included an average of 6.6 $\mathrm{H}^{27} \rightarrow p \Xi^{-} \rightarrow p\left(\Lambda \pi^{-}\right)$embedded decays per event (see following discussion). The cross-hatched region indicates the number of reconstructed $p-\pi^{-}$pairs actually produced by $\Lambda$ decays in the simulation. The reconstruction efficiency for primary $\Lambda$ s (i.e. number correctly reconstructed divided by number in acceptance) obtained here was about 5-6\%, 8 in reasonable agreement with the $8 \%$ value in Ref. [30] and the range of $4-15 \%$ in Ref. [20]. These comparisons verify that the FSDR $\Lambda$ simulation results presented here are consistent with those obtained from more detailed studies. The $\pm 3 \sigma$ width of the reconstructed $\Lambda$ peak in Fig. 2 is about $8 \mathrm{MeV}$ which quantitatively agrees with that found in Ref. [30]. Further cuts could reduce the background for primary $\Lambda \mathrm{s}$ as in Ref. [30], but this was not done here in order to retain the secondary $\Lambda$ s from $\Xi^{-}$decays.

A DCA cut $(4 \mathrm{~mm})$ among all pairs of selected $\Lambda$ candidates [i.e., those with impact parameter less than $2 \mathrm{~cm}$ from the primary vertex and which have a reconstructed mass within $\pm 3 \sigma( \pm 4 \mathrm{MeV})$ of the peak in Fig. 2] with all remaining

\footnotetext{
${ }^{8}$ The reconstruction efficiency for $\Lambda$ s from $\Xi^{-}$decays is somewhat higher than that for primary $\Lambda$ s since the decay vertices are distributed farther from the primary vertex where the proton and $\pi^{-}$daughters are not as likely to be removed by the $1 \mathrm{~mm}$ impact parameter cut as occurs for the daughter tracks from primary $\Lambda$ decays.
} 
secondary $\pi^{-}$s yielded the $\Xi^{-}$spectrum shown in Fig. 3 using the same events as in Fig. 2. The cross-hatched region indicates $\Lambda$ and $\pi^{-}$reconstructed pairs that originate from $\Xi^{-}$decays. The FSDR $\Xi^{-}$reconstruction efficiency with the preceding cuts was about 0.06 which is larger than that in Ref. [30]. The higher reconstruction efficiency obtained here is offset by the smaller peak signal-to-background ratio here $\left(\sim \frac{1}{4}\right)$ compared to the larger value $(\sim 1)$ in Ref. [30]. The $\pm 3 \sigma$ width of the reconstructed $\Xi^{-}$peak in Fig. 3 is about $10 \mathrm{MeV}$, in good agreement with Ref. [30]. We refer to the present set of cuts as "relaxed" while those in Ref. [30] are "tight." A tight selection of primary protons (impact parameter from primary vertex $\leq 100 \mu \mathrm{m}$ ) and $\Xi^{-}$candidates (within $\pm 6 \mathrm{MeV}$ of the peak in Fig. 3) with projected DCAs $\leq 6 \mathrm{~mm}$, provided the set of $p \Xi^{-}$pairs used in the invariant mass plot in Fig. 田.

Variable numbers of $\mathrm{H}^{27}$ resonances were randomly embedded in the HIJET [31] event generator output for $200 \mathrm{~A} \cdot \mathrm{GeV} \mathrm{Au+Au} \mathrm{central} \mathrm{collisions} \mathrm{according} \mathrm{to} \mathrm{the}$ following distribution,

$$
\frac{d N}{d p^{3}}=A \mathrm{e}^{-m_{T} / T} \mathrm{e}^{-\beta m_{T} \cosh (y)} .
$$

In Eq. (19) $m_{T}=\sqrt{M\left(H^{27}\right)^{2}+p_{T}^{2}}$ is the transverse mass, $T=238 \mathrm{MeV}, \beta=$ $3.0 \mathrm{GeV}^{-1}, A$ is a normalization constant, and at midrapidity $(y=0)$ the $m_{T}$ distribution corresponds to an effective temperature of $139 \mathrm{MeV}$. For this distribution function approximately $39 \%$ of the total $\mathrm{H}^{27}$ s have all four decay particles fully contained within the acceptance (Table [II). In the following discussion, production rates refer to the number of $\mathrm{H}^{27} \rightarrow p \Xi^{-}$decays per event for which all four decay particles are within the STAR acceptance.

In FSDR the $\mathrm{H}^{27}$ masses were randomly distributed according to the P-matrix resonance mass distribution (Sec. 3). The latter was obtained from the $N \Xi$ total elastic cross section in Eq. (3) by eliminating the non-resonant amplitude $\left(\bar{a}_{1}\right.$ was set to 0 ) and by removing the incident flux factor in the definition of the total cross section (i.e. by multiplying by $k_{1} / m$ ). The resonant $p \Xi^{-}$invariant mass probability distribution was therefore assumed to be

$$
P\left(M_{\mathrm{H}^{27}}\right)=P_{0} k_{1} \sigma_{\mathrm{TOT}}\left(\epsilon, \bar{a}_{1}=0\right) / m,
$$

where $M_{\mathrm{H}^{27}} \equiv \epsilon$ is the randomly sampled $\mathrm{H}^{27}$ mass and $P_{0}$ is a normalization constant.

In this work the $\mathrm{H}^{27}$ resonance energy, $\epsilon_{1}$, was assumed to be $2 \mathrm{MeV}$ above the $p \Xi^{-}$threshold and a range of width parameters, $\rho_{1}$, was assumed where $\rho_{1}=10$, 23, 37 and $50 \mathrm{MeV}$, corresponding to mass distributions with full width at half maximum (FWHM) values of $0.94,2.4,4.1$ and $5.6 \mathrm{MeV}$, respectively. Average $\mathrm{H}^{27}$ production rates $\left(n_{H}\right)$ of 3.1 (8 total) and 4.7 (12 total) per central $\mathrm{Au}+\mathrm{Au}$ HIJET event were assumed. For each of the resulting eight cases, which correspond to different values for $\rho_{1}$ and $n_{H}, 1940$ events were analyzed.

The preceding $\mathrm{H}^{27}$ production rates and number of events were selected in order to generate statistically significant resonance signals, with modest computing 
requirements, such that straightforward background subtraction and signal determination techniques would suffice. The analytical model in the preceding subsection, when supplemented with the present FSDR results, can be used to obtain estimates of STAR's detection sensitivity and data volume requirements for much smaller dibaryon resonance production rates.

The $p \Xi^{-}$invariant mass spectrum is shown in Fig. 1 for $1940 \mathrm{Au}+\mathrm{Au}$ central collision events for the case in which $\rho_{1}=23 \mathrm{MeV}(\mathrm{FWHM}=2.4 \mathrm{MeV})$ and $n_{H}=3.1$. The $\mathrm{H}^{27}$ peak is quite apparent for this case when the resonance occurs near threshold and the background is rapidly decreasing. The contributions of the large number of false $\Xi^{-} \mathrm{s}$ (see Fig. 3) are dispersed throughout the spectrum. Notice that momentum resolution effects do not degrade the $\mathrm{H}^{27}$ peak. Finite momentum resolution 28] for the final, reconstructed daughter protons and pions in the decay chain, dibaryon $\rightarrow p \Xi^{-} \rightarrow p\left(\Lambda \pi^{-}\right) \rightarrow p\left(p \pi^{-}\right) \pi^{-}$, results in about $10 \mathrm{MeV} / \mathrm{c}$ uncertainty in the $p \Xi^{-}$relative momentum. For this case, where FWHM is $2.4 \mathrm{MeV}$, such effects would only broaden the peak in the reconstructed $p \Xi^{-}$ invariant mass spectrum to about $2.6-2.9 \mathrm{MeV}$.

The uncorrelated $p \Xi^{-}$background distribution near threshold is proportional to $\sqrt{\epsilon-\epsilon_{t h 1}}$. Therefore, we assumed the threshold constrained model for the $p \Xi^{-}$ background distribution given by

$$
B_{\text {mod }}(x)=a_{1} \sqrt{x}+a_{2} x^{2}+a_{3} x^{3}+a_{4} x^{4}+a_{5} x^{5},
$$

where $x=\epsilon-\epsilon_{t h 1}$ and $a_{1}, a_{2}, \ldots a_{5}$ are parameters to be determined by chi-square fits. Excellent fits were obtained to the nonresonant backgrounds for all cases as exemplified by the fit shown in Fig. A by the solid line. The cross hatched portion of the spectrum in Fig. 4 indicates the reconstructed $p \Xi^{-}$pairs from actual $\mathrm{H}^{27}$ decays where the remaining background is seen to be in quantitative agreement with the fit.

The background subtracted peak is shown in the inset panel in Fig. 田. For this and all other cases the mass range of the resonance peak was taken from threshold to $7 \mathrm{MeV}$ above threshold. In addition, a lower cut-off of 20 counts per $1 \mathrm{MeV}$ bin (specific for the number of events used in this analysis) was applied to remove the residual background fluctuations which are apparent in the inset panel. The remaining counts in the $7 \mathrm{MeV}$ invariant mass range, which were above the 20 counts per bin cut-off, constitute the measured signal, $S_{F S D R}$. For this case the $\mathrm{H}^{27}$ reconstruction efficiency is 0.047 .

The results for each case are summarized in Table IV and in Fig. 5 which give the statistical significance of the signal, $(S / \Delta S)_{F S D R}$. The quantity $(S / \Delta S)_{F S D R}$ was calculated using

$$
\left(\frac{S}{\Delta S}\right)_{F S D R}=\frac{S_{F S D R}}{\sqrt{2 Y_{F S D R}-S_{F S D R}}},
$$

where $Y_{F S D R}$ is the total yield in the $7 \mathrm{MeV}$ wide domain of the resonance peak. Also listed in Table [V are estimates of the number of $\mathrm{Au}+\mathrm{Au}$ events which are 
needed in order to achieve $(S / \Delta S)_{F S D R}=3$, assuming statistical errors only, constant $f_{H-c u t}$, and using the $N_{e v}$ dependence from Eq. (15) to scale the FSDR results.

The decrease in $(S / \Delta S)_{F S D R}$ with increased resonance width and fixed production rate is due to increased signal losses $\left(i . e\right.$. reduced $f_{H-c u t}$ ) where, (1) more counts are lost in the tails of the resonance distribution above the upper mass limit, and (2) more counts are lost due to the 20 counts per bin cut-off. If the resonance mass domain was also increased, then $(S / \Delta S)_{F S D R}$ would further decrease due to the larger background contribution to $\Delta S$. From Table $\mathbb{D}$ and Fig. 5 it is seen that the required number of events to analyze in order to achieve a certain statistically significant signal $\left[\right.$ e.g. $\left.(S / \Delta S)_{F S D R}=3\right]$ increases for broader resonances and/or reduced production rates, as expected.

For analysis of real STAR data, it is likely that a more accurate background subtraction method will be needed than that used here, since optimistic production rates were assumed in this numerical study. A possible method is to form random $p \Xi^{-}$invariant mass histograms using mixed event pairs, normalized to the actual number of $p \Xi^{-}$pairs in the data. This removes dynamical correlations in the $p \Xi^{-}$ background spectra and is analogous to similar methods used successfully in pion interferometry analyses 32].

For smaller rates of production (e.g. $\left.n_{H} \approx 1\right)$ Eq. (16) simplifies to,

$$
N_{e v} \cong \frac{2(S / \Delta S)^{2} n_{t h, p} e_{p}\left(n_{t h, \Xi} e_{\Xi}+N_{F a l s e, \Xi}\right) F\left(\rho_{1}\right)}{\left(n_{H} e_{p} e_{\Xi} f_{H-c u t}\right)^{2}}
$$

where contributions from $\Delta \bar{S}_{\text {syst }}$ and $\bar{S}^{\prime}$ were neglected. The required number of events is proportional to $n_{H}^{-2}$ as expected [29]. For $n_{H}$ (total) $\approx 1$ and using the results in Table [V] it is estimated that of order $10^{4}-10^{5} \mathrm{Au}+\mathrm{Au}$ events must be analyzed to achieve a minimum dibaryon signal with $S / \Delta S=3$. At STAR, nominal run-time operations should obtain $10^{6}$ central $\mathrm{Au}+\mathrm{Au}$ events in about two weeks.

It is likely that the more crucial limiting factor in STAR's ability to detect dibaryon resonances, if they exist in the mass range considered here, will be due to systematic errors. Although the sources and magnitudes of these types of errors are not known at this time, a simple estimate can be made assuming the false counts due to systematic errors scale with overall yield. From Eq. (16) the minimum detectable signal is determined by the inequality in Eq. (17) provided $N_{e v}$ can be arbitrarily large. If we assume $\Delta \bar{S}_{\text {syst }}=f \bar{B}$, where $f$ is a constant, and use Eq. (7) with constant $f_{H-c u t}$ [29], the detectable range of dibaryon resonance production is given by

$$
n_{H}>(S / \Delta S) f \hat{n}_{H}(\bar{B} / \hat{\bar{S}})
$$

where $\hat{n}_{H}$ and $\hat{\bar{S}}$ refer to a specific simulation result. The $n_{H}($ total $)=8$ FSDR results indicate that $(\bar{B} / \bar{S}) \approx 3$. Requiring $S / \Delta S=3$ and assuming, for example, that the systematic errors are $1 \%$ of the background, the minimum, detectable 
dibaryon production rate is $n_{H}$ (total) $\approx 0.7$. Since STAR is a dedicated, long-term facility, discovery of dibaryon resonances of the type discussed here is well within reach of the experiment, provided the systematic errors can be kept small (to a few percent) and the dibaryon production rate is of order 1 per event or greater.

\section{Summary and Conclusions}

The possibility that $Y=0$ dibaryon states may occur as narrow resonances, if located in energy just above the strong interaction breakup threshold, offers interesting new discovery opportunities for the relativistic heavy ion physics program at RHIC, especially for the STAR experiment. In the baryon octet $\otimes$ octet direct product space for $Y=0$ dibaryons we have listed eight possible states (corresponding to $\mathrm{I}_{3}=0$ ) which could, in principle, be discovered by STAR with its SVT-SSD central vertex tracking system. If produced, these $I_{3}=0$ states decay to $\Lambda \Lambda$ and/or $p \Xi^{-}$channels, depending on the resonance energy. In addition, the formation of these resonances may be significantly enhanced in a color-deconfined medium, such as the QGP, by way of the dominant color-octet-octet component of the dibaryon wave function. A significant change in the production rate of these states, in conjunction with other QGP signals, would provide strong corroborative evidence for QGP formation. High quality statistics for the $p \Xi^{-}$invariant mass spectrum can be expected at STAR with the analysis of data from of order several hundred thousand central $\mathrm{Au}+\mathrm{Au}$ collision events - several days of STAR data acquisition.

One specific $Y=0$ dibaryon state was used to illustrate and estimate the detection and sensitivity range of STAR. This state, the $J^{\pi}=0^{+}, \mathrm{I}=1, \mathrm{I}_{3}=0$ member of the 27-plet, was assumed to lie within a few $\mathrm{MeV}$ above the $p \Xi^{-}$ strong breakup threshold. The resonance distribution was estimated using the Pmatrix formalism [9], and the detector response and event reconstruction/analysis effects were estimated using a fast simulation, detector response code. This new simulation tool, FSDR, enables rapid evaluation of relativistic heavy ion detector/reconstruction/analysis capabilities for proposed, new physics programs at RHIC.

The ability of the STAR experiment to detect a possible $\mathrm{H}^{27} \rightarrow p \Xi^{-}$dibaryon resonance decay was evaluated in terms of the statistical significance of the dibaryon signal as a function of the $\mathrm{H}^{27}$ production rate and width (FWHM). The numerical simulation results, together with an analytical model, were used to estimate the data volume requirements for much smaller dibaryon resonance production rates. Improved background subtraction methods were suggested for the case of reduced production rates; limitations due to systematic errors were also estimated. The large data volume required (of order $10^{6}$ central events) for rare particle searches of the type discussed here is compatible with the nominal run plan for STAR. The discovery of possible dibaryon resonances with widths of order a few $\mathrm{MeV}$ which lie just above their strong decay threshold is feasible at STAR, provided systematic errors are small (few percent) and the dibaryon resonance production rate is of 
order 1 or greater per $\mathrm{Au}+\mathrm{Au}$ central collision.

\section{Acknowledgements}

We thank Rene Bellwied, Helen Caines, John Harris, Peter Jones and Craig Ogilvie for their comments and careful review of the manuscript. The support of the STAR Silicon Vertex Tracker and Strangeness Physics Working groups is acknowledged. This research was supported in part by The U. S. Department of Energy Grant DE-FG03-94ER40845 and The Robert A. Welch Foundation Grant F-604. 


\section{References}

[1] Particle Data Group, Eur. Phys. J. C 3, 1 (1998); see pgs. 609-611.

[2] R. L. Jaffe, Phys. Rev. Lett. 38, 195 (1977); erratum ibid. 38, 617 (1977).

[3] T. Sakai, K. Shimizu and K. Yasaki, eprint Archive nucl-th/9912063, submitted to Prog. Theo. Phys. Suppl. (Jan., 2000), unpublished.

[4] R. Klingenberg, J. Phys. G: Nucl. Part. Phys. 25, R273 (1999).

[5] D. P. Goyal, J. N. Misra and A. V. Sodhi, Phys. Rev. D 21, 607 (1980); S. Aoki et al., Phys. Rev. Lett. 65, 1729 (1990); S. Aoki et al., Prog. Theor. Phys. 85, 1287 (1991); K. Imai, Nucl. Phys. A 527, 181c (1991); J. K. Ahn et al., Phys. Lett. B 378, 53 (1996); R. W. Stotzer et al., Phys. Rev. Lett. 78, 3646 (1997); P. Beillière et al., Phys. Lett. B 39, 671 (1972); G. Wilquet, W. L. Knight, J. G. Guy, S. N. Tovey, J. Canter, A. Mann, J. Schneps, G. Wolsky, D. J. Miller and F. R. Stannard, Phys. Lett. B 57, 97 (1975).

[6] B. A. Shahbazian, V. A. Sashin, A. O. Kechechyan and A. S. Martynov, Phys. Lett. B 235, 208 (1990).

[7] J. Belz et al., Phys. Rev. Lett. 76, 3277 (1996).

[8] R. J. Oakes, Phys. Rev. 131, 2239 (1963); A. Th. M. Aerts, P. J. G. Mulders and J. J. de Swart, Phys. Rev. D 17, 260 (1978); J. F. Donoghue, E. Golowich and B. R. Holstein, Phys. Rev. D 34, 3434 (1986); S.-Q. Xie, J. Phys. G Nucl. Part. Phys. 15, 287 (1989); and R. L. Jaffe, private communication.

[9] S. V. Bashinsky and R. L. Jaffe, Nucl. Phys. A 625, 167 (1997).

[10] J. W. Negele, A. Pochinsky and B. Scarlet, Nucl. Phys. B (Proc. Suppl.) 73, 255 (1999); I. Wetzorke, F. Karsch and E. Laermann, eprint Archive heplat/9909037, poster presented at the $17^{\text {th }}$ International Symp. on Lattice Field Theory (LATTICE 99), Pisa, Italy (1999).

[11] R. L. Jaffe and C. L. Korpa, Nucl. Phys. B 258, 468 (1985); U. Straub, Z.Y. Zhang, K. Bräuer, A. Faessler and S. B. Khadkikar, Phys. Lett. B 200, 241 (1988); Y. Iwasaki, T. Yoshie and Y. Tsuboi, Phys. Rev. Lett. 60, 1371 (1988); M. Oka, Phys. Rev. D 38, 298 (1988); Y. Koike, K. Shimizu and K. Yazaki, Nucl. Phys. A 513, 653 (1990).

[12] R. D. Cousins and J. R. Klein, Phys. Rev. D 56, 1673 (1997).

[13] C. B. Dover, P. Koch and M. May, Phys. Rev. C 40, 115 (1989).

[14] J. Schaffner-Bielich, R. Mattiello and H. Sorge, e-Print Archive nuclth/9908043, unpublished, (1999). 
[15] D. E. Kahana and S. H. Kahana, Phys. Rev. C 60, 065206 (1999).

[16] A. Jacholkowski et al., WA97 Collaboration, J. Phys. G: Nucl. Part. Phys. 25, 423 (1999).

[17] J. K. Ahn et al. (KEK-PS E224 Collaboration), Nucl. Phys. A 639, 379c (1998); Phys. Lett. B 444, 267 (1998).

[18] R. P. Bickerstaff and B. G. Wybourne, J. Phys. G: Nucl. Phys. 7, 275 (1981).

[19] STAR Collaboration, J. W. Harris et al., Nucl. Phys. A 566, 277c (1994); J. W. Harris and B. Müller, Annu. Rev. Nucl. Part. Sci. 46, 71 (1996); STAR Collaboration, "Conceptual Design Report for the Solenoidal Tracker at RHIC," Lawrence Berkeley Laboratory Publication PUB-5347 (1992), unpublished.

[20] A. Boucham et al., "Proposal for a Silicon Strip Detector for STAR," (1999), unpublished.

[21] J. P. Coffin and C. Kuhn, J. Phys. G: Nucl. Part. Phys. 23, 2117 (1997).

[22] C. Pigot et al., Nucl. Phys. B 249, 172 (1985).

[23] H. Piekarz, Nucl. Phys. A 450, 85c (1986).

[24] C. B. Dover and H. Feshbach, Ann. Phys. (N. Y.) 198, 321 (1990).

[25] N. Kodama, M. Oka and T. Hatsuda, Nucl. Phys. A 580, 445 (1994).

[26] S. D. Paganis, T. Udagawa, G. W. Hoffmann and R. L. Ray, Phys. Rev. C 56, 570 (1997).

[27] R. G. Newton, "Scattering Theory of Waves and Particles," (McGraw-Hill, New York, 1966), pgs. 528-539; L. Fonda and R. G. Newton, Nuovo Cimento 14, 1027 (1959).

[28] R. Bellwied (for the STAR Collaboration), "The Measurement of Hadronic Observables with the Solenoidal Tracker at RHIC (STAR)," in Proceedings of the Pre-Conference Workshop, Eleventh International Conference on UltraRelativistic Nucleus-Nucleus Collisions - Quark Matter '95, Monterey, CA, USA, eds. by J. Thomas and T. Hallman, University of California Publication UCRL-ID-121571 (1995), p. 41.

[29] In general $f_{H-c u t}$ depends on $n_{H}, N_{e v}, \rho_{1}$, etc. For example, the number of lost signal counts due to the background fluctuation cut depends on the size of the statistical fluctuations in the background subtracted spectrum which is proportional to $\sqrt{\bar{B} N_{e v}}$. Refering to quantities in Sec. 4.1, $f_{H-c u t} \equiv S / S_{T} \equiv$ 
$\left(S_{T}-S^{\prime}\right) / S_{T} \cong\left(n_{H} e_{p} e_{\Xi} N_{e v}-C \sqrt{\bar{B} N_{e v}}\right) /\left(n_{H} e_{p} e_{\Xi} N_{e v}\right)$, where $C$ depends on the invariant mass domain size, resonance width, and bin size. $\bar{S}$ and $\bar{S}^{\prime}$ in general depend on $N_{e v}$. In this work we consider two kinds of applications in which $f_{H-c u t}$ can be regarded as constant with respect to $n_{H}$ and $N_{e v}$ : (1) strong $\mathrm{H}^{27}$ signals where $f_{H-c u t} \approx 1$, and $(2)$ weak $\mathrm{H}^{27}$ signals where $N_{e v} \propto n_{H}^{-2}$ is required.

[30] R. Bellwied, J. Phys. G: Nucl. Part. Phys. 25, 437 (1999); W. K. Wilson (for the STAR Collaboration), "Measurements of Strangeness Production in the STAR Experiment at RHIC," in Proceedings of the Pre-Conference Workshop, Eleventh International Conference on Ultra-Relativistic NucleusNucleus Collisions - Quark Matter '95, Monterey, CA, USA, eds. by J. Thomas and T. Hallman, University of California Publication UCRL-ID121571 (1995), p. 55.

[31] T. Ludlam, A. Pfoh and A. Shor, RHIC Workshop I, eds. P. Haustein and C. Woody (Brookhaven, April 1985), BNL-51921; A. Shor and R. Longacre, Phys. Lett. B 218, 100 (1989).

[32] I. G. Bearden et al., NA44 Collaboration, Phys. Rev. C 58, 1656 (1998); H. Appelshäuser et al., NA49 Collaboration, Eur. Phys. J. C 2, 661 (1998). 
Table I: $J^{\pi}=0^{+}, 1^{+} \quad Y=0$ dibaryon constituents of the $\mathbf{8} \otimes \mathbf{8}$ baryon octet baryon octet direct product space for each irreducible $\mathrm{SU}(3)$ flavor representation. The dominant decay modes for each resonance mass range are shown. NN abbreviates "mass of nucleon + nucleon," and similarly for the other baryon-baryon pairs. Weak decays which reduce the number of strange quarks by 1 or 2 are denoted by " $\Delta S=1$ " or " $\Delta S=2$," respectively. "S" denotes strong interaction decays. "EM" denotes electromagnetic decays. "S-Iso" indicates strong interaction decays via the small isospin admixtures in the physical states. " $\mathrm{S}$ " indicates that strong interaction decays from the $\mathrm{I}=1$ states are possible to both $N \Xi$ and $\Lambda \Sigma$.

\begin{tabular}{|c|c|c|c|c|c|c|c|c|}
\hline \multirow{2}{*}{$\begin{array}{c}\overline{\mathrm{SU}}(3)_{f} \\
\text { Irrep. }\end{array}$} & \multirow[b]{2}{*}{$\left(J^{\pi}, \mathrm{I}, \mathrm{I}_{3}\right)$} & \multicolumn{7}{|c|}{ Resonance Mass Range } \\
\hline & & NN-N $\Lambda$ & $\mathrm{N} \Lambda-\mathrm{N} \Sigma$ & $\mathrm{N} \Sigma-\Lambda \Lambda$ & $\Lambda \Lambda-N \Xi$ & $N \Xi-\Lambda \Sigma$ & $\Lambda \Sigma-\Sigma \Sigma$ & $>\Sigma \Sigma$ \\
\hline 1 & $0^{+} 00$ & $\Delta S=2$ & $\Delta S=1$ & $\Delta S=1$ & S & $\mathrm{S}$ & $\mathrm{S}$ & $\mathrm{S}$ \\
\hline 8 & $1^{+} 00$ & $\Delta \mathrm{S}=2$ & $\Delta S=1$ & $\Delta \mathrm{S}=1$ & EM & S & $\mathrm{S}$ & $\mathrm{S}$ \\
\hline 8 & $1^{+} 11$ & $\Delta S=2$ & $\Delta S=1$ & $\Delta S=1$ & $\Delta S=1$ & S & S & $\mathrm{S}$ \\
\hline 8 & $1^{+} 10$ & $\Delta S=2$ & $\Delta S=1$ & $\Delta S=1$ & EM & $\mathrm{S}$ & $\mathrm{S}$ & $\mathrm{S}$ \\
\hline 8 & $1^{+} 1-1$ & $\Delta S=2$ & $\Delta \mathrm{S}=1$ & $\Delta \mathrm{S}=1$ & $\Delta S=1$ & S & S & $\mathrm{S}$ \\
\hline 10 & $1^{+} 11$ & $\Delta S=2$ & $\Delta S=1$ & $\Delta S=1$ & $\Delta S=1$ & $\mathrm{~S}$ & $\mathrm{~S}^{\prime}$ & $\mathrm{S}$ \\
\hline 10 & $1^{+} 10$ & $\Delta S=2$ & $\Delta S=1$ & $\Delta S=1$ & EM & S & $\mathrm{S}^{\prime}$ & S \\
\hline 10 & $1^{+} 1-1$ & $\Delta S=2$ & $\Delta S=1$ & $\Delta S=1$ & $\Delta S=1$ & S & $S^{\prime}$ & S \\
\hline$\overline{\mathbf{1 0}}$ & $1^{+} 11$ & $\Delta S=2$ & $\Delta S=1$ & $\Delta S=1$ & $\Delta S=1$ & S & $\mathrm{S}^{\prime}$ & S \\
\hline$\overline{\mathbf{1 0}}$ & $1^{+} 10$ & $\Delta \mathrm{S}=2$ & $\Delta S=1$ & $\Delta S=1$ & EM & S & $\mathrm{S}^{\prime}$ & $\mathrm{S}$ \\
\hline$\overline{\mathbf{1 0}}$ & $1^{+} 1-1$ & $\Delta S=2$ & $\Delta S=1$ & $\Delta S=1$ & $\Delta S=1$ & S & $\mathrm{S}^{\prime}$ & S \\
\hline 27 & $0^{+} 00$ & $\Delta S=2$ & $\Delta S=1$ & $\Delta S=1$ & S & S & S & S \\
\hline 27 & $0^{+} 11$ & $\Delta S=2$ & $\Delta S=1$ & $\Delta S=1$ & $\Delta S=1$ & S & $\mathrm{S}^{\prime}$ & S \\
\hline 27 & $0^{+} 10$ & $\Delta S=2$ & $\Delta S=1$ & $\Delta S=1$ & $\begin{array}{c}\mathrm{EM}, \\
\mathrm{S}-\mathrm{IsO}\end{array}$ & $\mathrm{S}$ & $S^{\prime}$ & $S$ \\
\hline 27 & $0^{+} 1-1$ & $\Delta S=2$ & $\Delta S=1$ & $\Delta S=1$ & $\Delta S=1$ & $\mathrm{~S}$ & $\mathrm{~S}^{\prime}$ & $\mathrm{S}$ \\
\hline 27 & $0^{+} 22$ & $\Delta S=2$ & $\Delta S=1$ & $\Delta \mathrm{S}=1$ & $\Delta S=1$ & $\Delta S=1$ & $\Delta S=1$ & S \\
\hline 27 & $0^{+} 21$ & $\Delta S=2$ & $\Delta S=1$ & $\Delta S=1$ & $\Delta S=1$ & $\mathrm{~S}-\mathrm{EM}$, & $\mathrm{S}-\stackrel{\mathrm{EM},}{\mathrm{ISO}}$ & $\mathrm{S}$ \\
\hline 27 & $0^{+} 20$ & $\Delta S=2$ & $\Delta S=1$ & $\Delta S=1$ & $\begin{array}{l}\text { EM, } \\
\mathrm{S}-\text { Iso }\end{array}$ & $\mathrm{S}-\mathrm{EM}$, Iso & $\begin{array}{c}\text { EM, } \\
\mathrm{S}-\mathrm{Iso}\end{array}$ & S \\
\hline 27 & $0^{+} 2-1$ & $\Delta S=2$ & $\Delta S=1$ & $\Delta S=1$ & $\Delta S=1$ & $\mathrm{~S}-\mathrm{EM}$, & $\begin{array}{c}\text { EM, } \\
\mathrm{S}-\mathrm{ISO}\end{array}$ & $\mathrm{S}$ \\
\hline 27 & $0^{+} 2-2$ & $\Delta S=2$ & $\Delta S=2$ & $\Delta \mathrm{S}=1$ & $\Delta S=1$ & $\Delta S=1$ & $\Delta S=1$ & S \\
\hline
\end{tabular}


Table II: Optimum decay channels and resonance mass ranges for $J^{\pi}=0^{+}, 1^{+} Y=$ 0 dibaryon resonance searches.

\begin{tabular}{|c|c|c|c|}
\hline $\begin{array}{c}\mathrm{SU}(3)_{f} \\
\text { Irrep. }\end{array}$ & $\left(J^{\pi}, \mathrm{I}, \mathrm{I}_{3}\right)$ & Decay Channels and Resonance Mass Ranges (MeV) & $\begin{array}{c}\text { Accessible } \\
\text { to STAR }\end{array}$ \\
\hline 1 & $0^{+} 00$ & $\Lambda \Lambda \gtrsim 2231$ & Yes \\
\hline 8 & $1^{+} 00$ & $\Lambda \Lambda \gtrsim 2231 ; n \Xi^{0} \gtrsim 2254 ; p \Xi^{-} \gtrsim 2260$ & Yes \\
\hline 8 & $1^{+} 11$ & $p \Xi^{0} \gtrsim 2253$ & \\
\hline 8 & $1^{+} 10$ & $\Lambda \Lambda \gtrsim 2231 ; n \Xi^{0} \gtrsim 2254 ; p \Xi^{-} \gtrsim 2260$ & Yes \\
\hline 8 & $1^{+} 1-1$ & $n \Xi^{-} \gtrsim 2261$ & \\
\hline 10 & $1^{+} 11$ & $p \Xi^{0} \gtrsim 2253$ & \\
\hline 10 & $1^{+} 10$ & $\Lambda \Lambda \gtrsim 2231 ; n \Xi^{0} \gtrsim 2254 ; p \Xi^{-} \gtrsim 2260$ & Yes \\
\hline 10 & $1^{+} 1-1$ & $n \Xi^{-} \gtrsim 2261$ & \\
\hline$\overline{\mathbf{1 0}}$ & $1^{+} 11$ & $p \Xi^{0} \gtrsim 2253$ & \\
\hline$\overline{\mathbf{1 0}}$ & $1^{+} 10$ & $\Lambda \Lambda \gtrsim 2231 ; n \Xi^{0} \gtrsim 2254 ; p \Xi^{-} \gtrsim 2260$ & Yes \\
\hline$\overline{\mathbf{1 0}}$ & $1^{+} 1-1$ & $n \Xi^{-} \gtrsim 2261$ & \\
\hline 27 & $0^{+} 00$ & $\Lambda \Lambda \gtrsim 2231$ & Yes \\
\hline 27 & $0^{+} 11$ & $p \Xi^{0} \gtrsim 2253$ & \\
\hline 27 & $0^{+} 10$ & $\Lambda \Lambda$ from 2231 to $2254 ; \rrbracket n \Xi^{0} \gtrsim 2254 ; p \Xi^{-} \gtrsim 2260$ & Yes \\
\hline 27 & $0^{+} 1-1$ & $n \Xi^{-} \gtrsim 2261$ & \\
\hline 27 & $0^{+} 22$ & $\Sigma^{+} \Sigma^{+} \gtrsim 2379$ & \\
\hline 27 & $0^{+} 21$ & $p \Xi^{0}$ from 2253 to $2382 ;$; $\Sigma^{+} \Sigma^{0} \gtrsim 2382$ & \\
\hline 27 & $0^{+} 20$ & $\Lambda \Lambda$ from 2231 to $2385 ;^{a} \Sigma \Sigma \gtrsim 2385$ & Yes' \\
\hline 27 & $0^{+} 2-1$ & $n \Xi^{-}$from 2261 to $2390 ; \Sigma^{0} \Sigma^{0} \Sigma^{-} \gtrsim 2390$ & \\
\hline 27 & $0^{+} 2-2$ & $\Sigma^{-} \Sigma^{-} \gtrsim 2395$ & \\
\hline
\end{tabular}

${ }^{a}$ If strong decay via isospin admixture dominates EM decay and resonance remains narrow; otherwise $\Lambda \Lambda$ for resonance mass $\gtrsim 2231 \mathrm{MeV}$ only.

${ }^{b}$ If strong decay via isospin admixture dominates EM decay and resonance remains narrow; otherwise $p \Xi^{0}$ for resonance mass $\gtrsim 2253 \mathrm{MeV}$ only.

${ }^{c} \Lambda \Lambda$ decay channel only.

${ }^{d}$ If strong decay via isospin admixture dominates EM decay and resonance remains narrow; otherwise $n \Xi^{-}$for resonance mass $\gtrsim 2261 \mathrm{MeV}$ only. 
Table III: Kinematic acceptance and reconstruction parameters assumed in the analytical and numerical simulations.

\begin{tabular}{|l|l|}
\hline Parameter & Value \\
\hline Transverse momentum acceptance & $p_{T}>0.1 \mathrm{GeV} / \mathrm{c}$ \\
Pseudorapidity acceptance & $|\eta| \leq 1$ \\
Azimuthal acceptance & $\phi=0 \rightarrow 2 \pi$ \\
Momentum resolution, $\Delta p / p$ & $2 \%$ \\
TPC track reconstruction efficiency ${ }^{\natural}$ & $90 \%$ \\
SVT track reconstruction efficiency & $80 \%$ \\
Particle Identification $(\pi$, protons $)$ & $100 \% 9$ \\
\hline
\end{tabular}

\footnotetext{
${ }^{a}$ Time Projection Chamber; main tracking detector for STAR.

${ }^{b}$ Silicon Vertex Tracker and Silicon Strip Detector tracking system for STAR.

${ }^{c}$ Actual value not yet available.
} 
Table IV: FSDR results for the statistical significance $(S / \Delta S)_{F S D R}$ of the $\mathrm{H}^{27} \rightarrow$ $p \Xi^{-}$dibaryon resonance decay signal for total production rates per $\mathrm{Au}+\mathrm{Au}$ central event of 8 and 12 and for four assumed values of resonance width.

\begin{tabular}{|cccccc|}
\hline $\begin{array}{c}n_{H} \\
\text { (Total) }\end{array}$ & $n_{H} \square^{\text {g }}$ & $\begin{array}{c}\rho_{1} \\
(\mathrm{MeV})\end{array}$ & $\begin{array}{c}\text { FWHM } \\
(\mathrm{MeV})\end{array}$ & $(S / \Delta S)_{F S D R}$ 向 & $\begin{array}{c}N_{\text {ev }} \text { for } \\
(S / \Delta S)_{F S D R}=39\end{array}$ \\
\hline 8 & 3.1 & 10 & 0.94 & 7.9 & 280 \\
8 & 3.1 & 23 & 2.4 & 6.4 & 426 \\
8 & 3.1 & 37 & 4.1 & 5.6 & 557 \\
8 & 3.1 & 50 & 5.6 & 4.1 & 1039 \\
\hline 12 & 4.7 & 10 & 0.94 & 10.2 & 168 \\
12 & 4.7 & 23 & 2.4 & 9.1 & 211 \\
12 & 4.7 & 37 & 4.1 & 8.6 & 236 \\
12 & 4.7 & 50 & 5.6 & 7.5 & 310 \\
\hline
\end{tabular}

${ }^{a}$ Number of $\mathrm{H}^{27} \rightarrow p \Xi^{-}$decays per event in which all decay products enter the STAR detector acceptance. This is $39 \%$ of the total for the assumed $\mathrm{H}^{27}$ momentum distribution model.

${ }^{b}$ For $1940 \mathrm{Au}+\mathrm{Au}$ central collision events.

${ }^{c}$ Assuming $(S / \Delta S)_{F S D R}$ is proportional to $\sqrt{N_{e v}}$ as discussed in the text. 


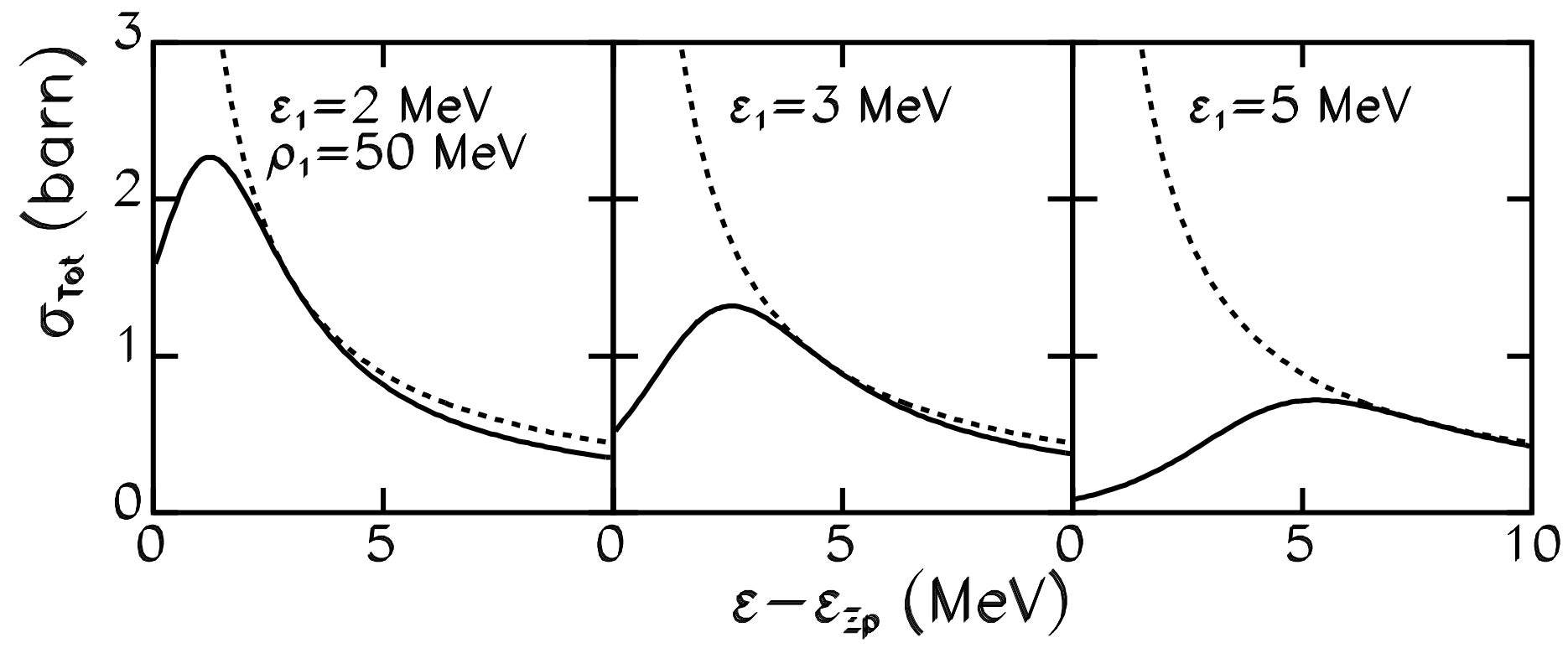

Figure 1: Total elastic cross sections for $N+\Xi$ scattering assuming the $\mathrm{H}^{27}$ dibaryon resonance is 2,3 and $5 \mathrm{MeV}$ above threshold are shown in the left, center and right panels, respectively. The dashed lines indicate the unitarity limit, $4 \pi / k_{1}^{2}$. The $N \Xi$ threshold energy is denoted by $\varepsilon_{\Xi p}$ (axis label). 


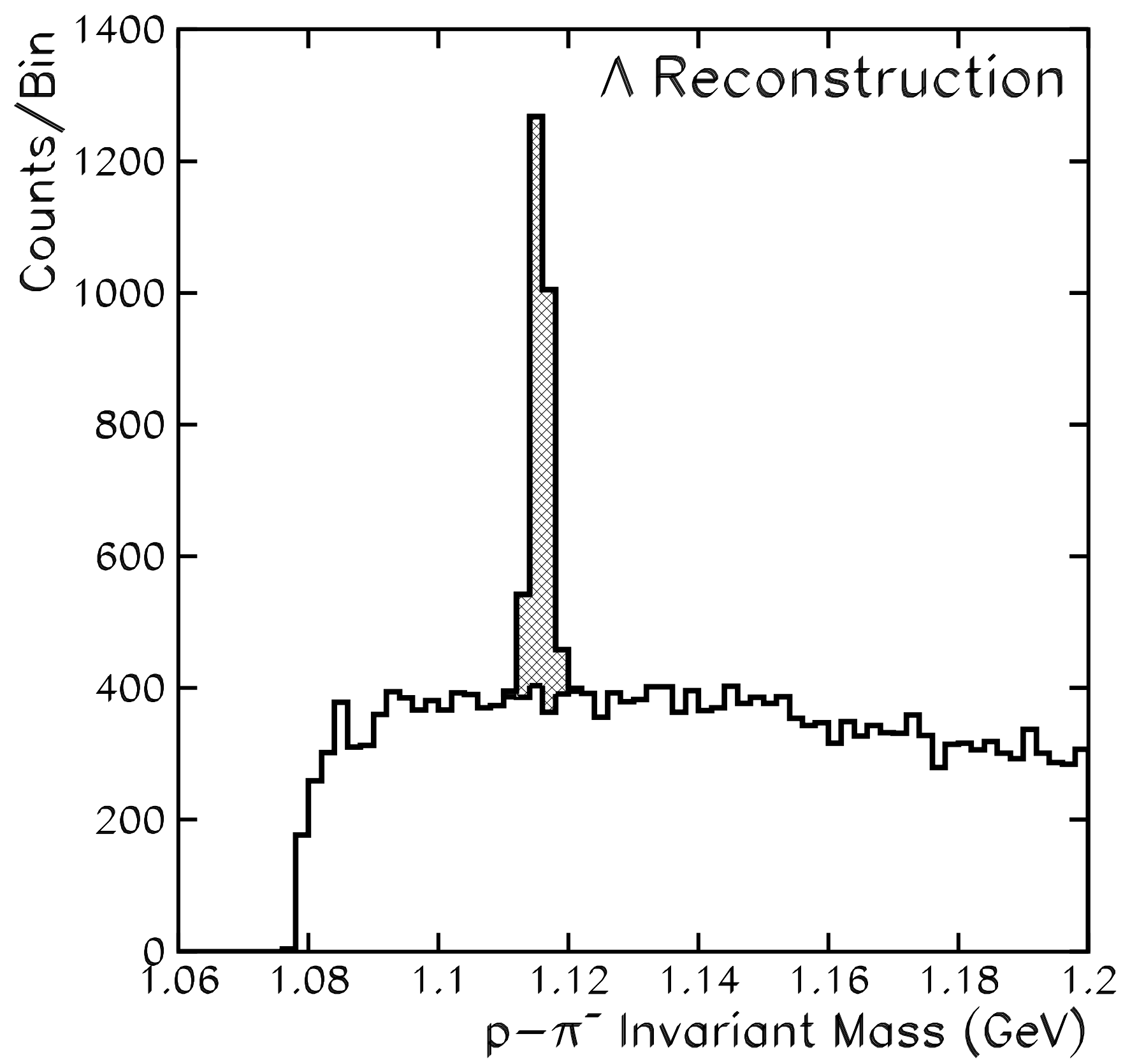

Figure 2: FSDR $p \pi^{-}$invariant mass spectrum for $\Lambda \rightarrow p \pi^{-}$reconstruction for 600 central $\mathrm{Au}+\mathrm{Au}$ HIJET events with embedded $\mathrm{H}^{27}$ resonances at a collision energy of $200 \mathrm{~A} \cdot \mathrm{GeV}$ as described in the text. The cross-hatched portion indicates the number of correctly reconstructed $\Lambda \rightarrow p \pi^{-}$decays. Bin size is $2 \mathrm{MeV}$. 


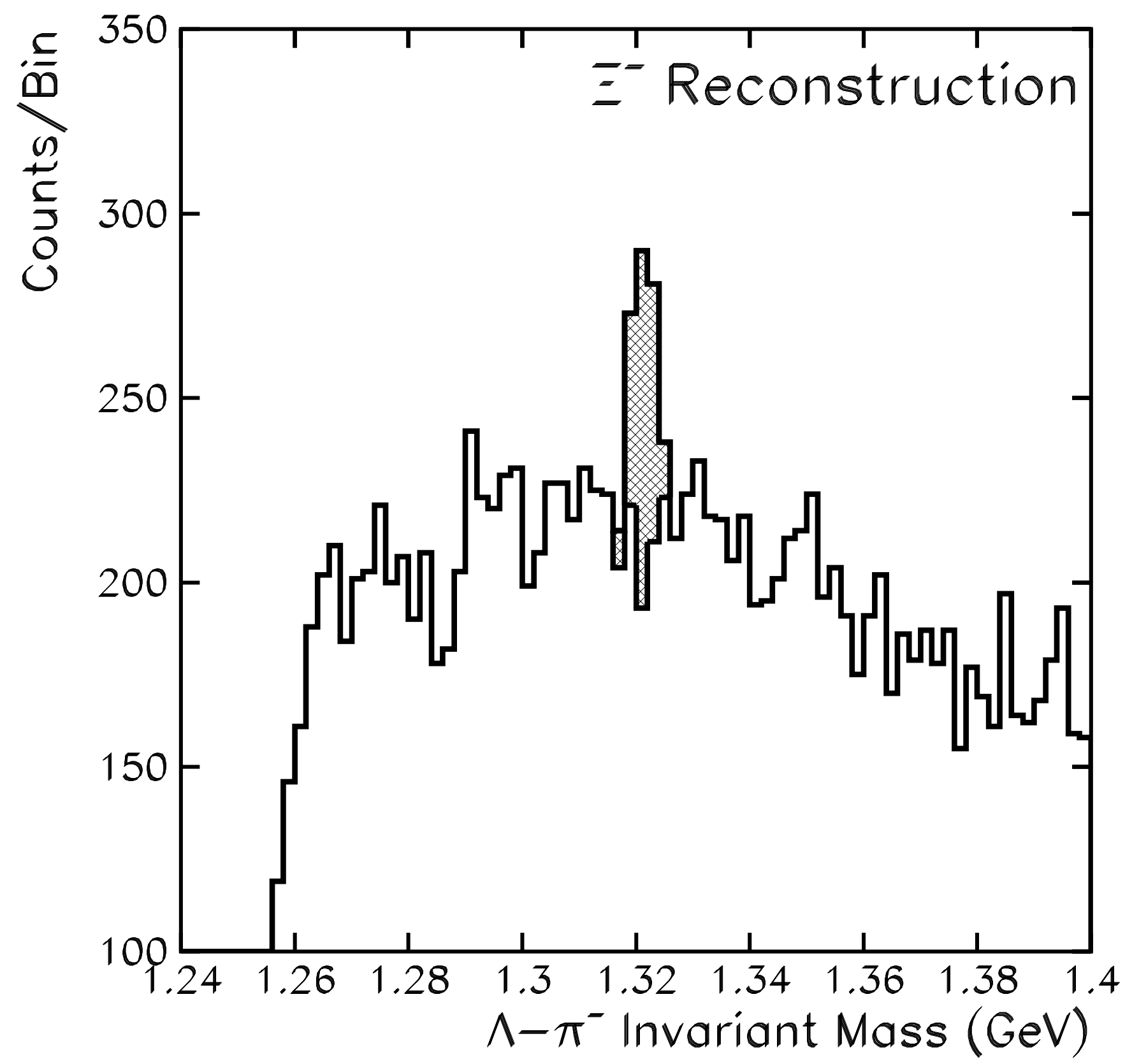

Figure 3: FSDR $\Lambda \pi^{-}$invariant mass spectrum for $\Xi^{-} \rightarrow \Lambda \pi^{-}$reconstruction for 600 central $\mathrm{Au}+\mathrm{Au}$ HIJET events with embedded $\mathrm{H}^{27}$ resonances at a collision energy of $200 \mathrm{~A} \cdot \mathrm{GeV}$, using the relaxed $\Xi^{-}$decay vertex reconstruction cuts described in the text. The cross-hatched portion indicates the number of correctly reconstructed $\Xi^{-} \rightarrow \Lambda \pi^{-}$decays. Bin size is $2 \mathrm{MeV}$. Note the suppressed zero for the vertical scale. 


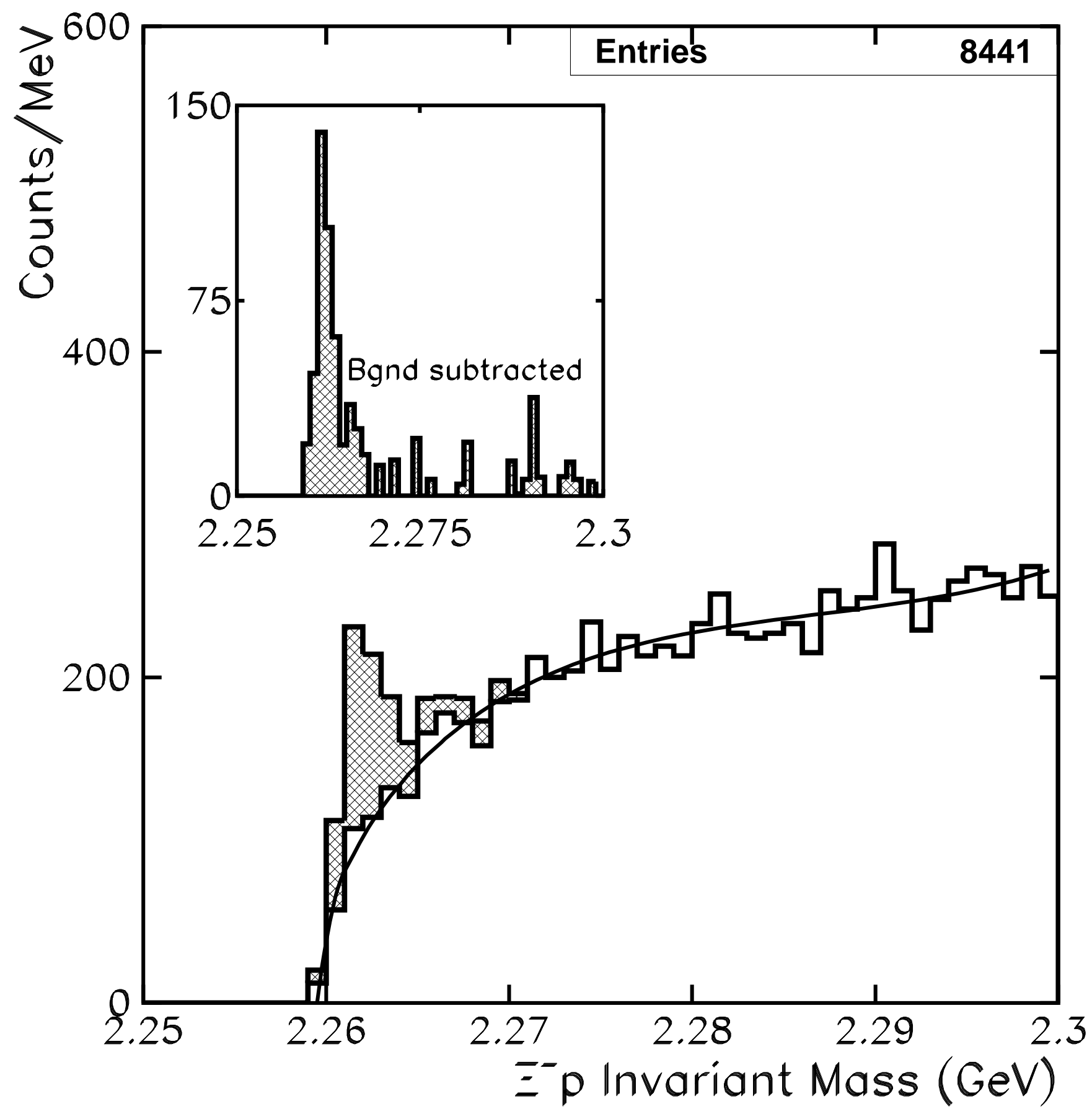

Figure 4: FSDR $p \Xi^{-}$invariant mass spectrum for $\mathrm{H}^{27} \rightarrow p \Xi^{-}$reconstruction for 1940 central $\mathrm{Au}+\mathrm{Au}$ HIJET events at a collision energy of $200 \mathrm{~A} \cdot \mathrm{GeV}$, assuming an average of $3.1 \mathrm{H}^{27} \rightarrow p \Xi^{-}$resonance decays per event into the acceptance with resonance energy $2 \mathrm{MeV}$ above threshold and P-matrix width parameter $\left(\rho_{1}\right)$ of $23 \mathrm{MeV}(\mathrm{FWHM}=2.4 \mathrm{MeV})$. The cross-hatched portion indicates the number of correctly reconstructed $\mathrm{H}^{27} \rightarrow p \Xi^{-}$decays. The background subtracted peak is shown in the inset panel. Bin size is $1 \mathrm{MeV}$. Solid curve indicates the five-term threshold constrained background model fit. 


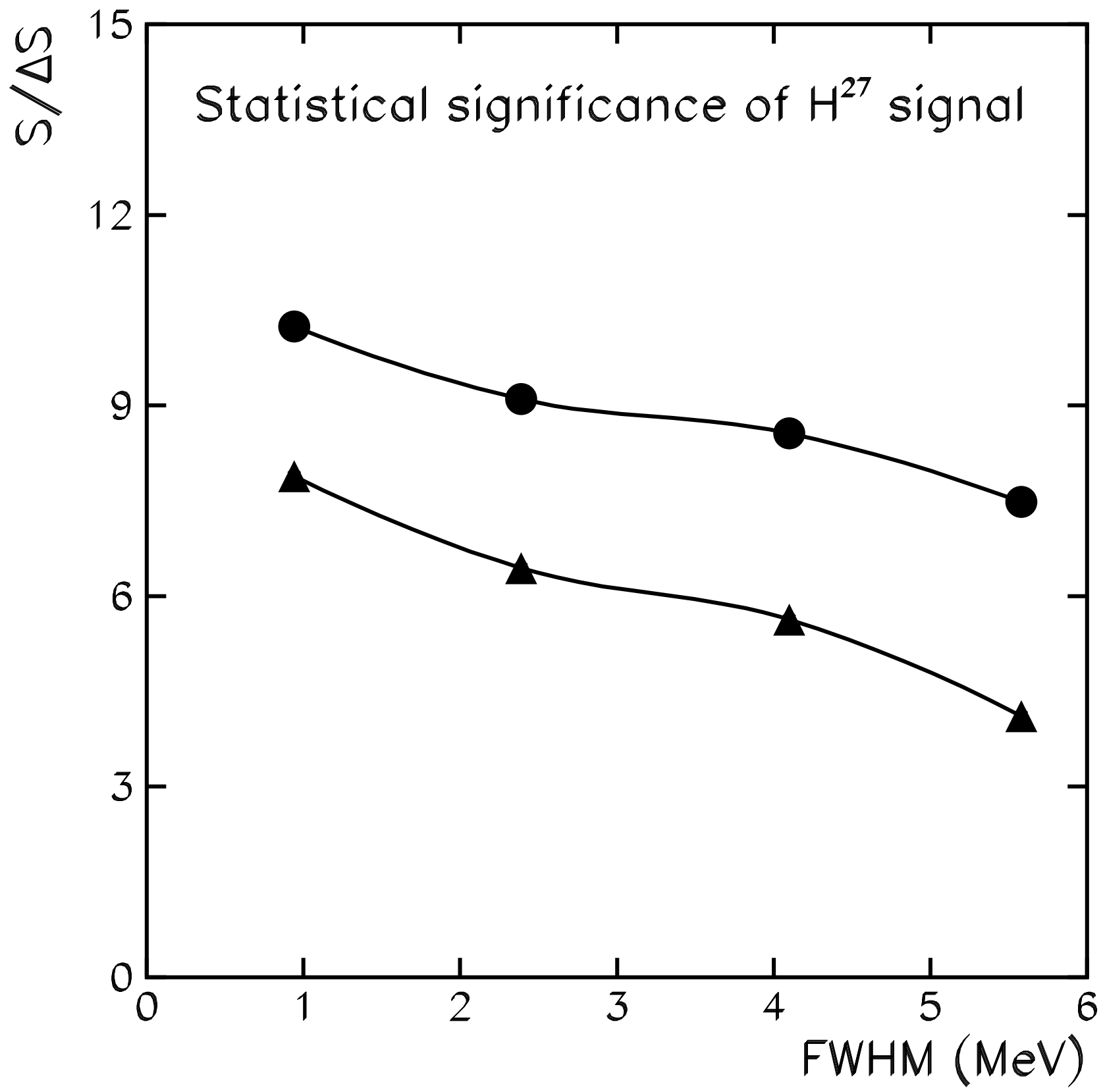

Figure 5: Statistical significance of the $\mathrm{H}^{27} \rightarrow p \Xi^{-}$dibaryon resonance decay signal as a function of resonance width (FWHM) for resonance production rates of 3.1 (triangles) and 4.7 (dots) per event (all decay products enter detector acceptance). For each case $1940 \mathrm{Au}+\mathrm{Au}$ central collisions were analyzed. Solid lines connect the values for fixed production rates. 